\title{
Correction: 8 A prospective longitudinal follow up study using T1 and T2 mapping sequences and twelve-segment myocardial assessment to identify and monitor myocardial inflammation in myositis
}

Bromage D, Nabeebaccus A, O'gallagher K, et al. 8 A prospective longitudinal follow up study using T1 and T2 mapping sequences and twelve-segment myocardial assessment to identify and monitor myocardial inflammation in myositis. Heart 2019;105:A7-8. doi: 10.1136/ heartjnl-2019-BSCMR.8.

In this abstract, the final author list should include Luke Dancy who should be appearing as the first author. There are two figures which should have been published with the abstract which were omitted. These have been included below.

\section{Background}

Myositis is a systemic autoimmune condition causing skeletal muscle inflammation and fibrosis as well as extra skeletal involvement. Cardiac involvement is well described and has morbidity and mortality. It can be diffuse or focal which can make assessment using conventional imaging techniques more difficult. To date the incidence is poorly defined using conventional cardiac investigations.

\section{Purpose}

To investigate the reproducibility of a 12 segment ventricular myocardial model of T1 and T2 mapping in healthy volunteers and then to use this methodology in patients with myositis, comparing it to the current non invasive gold standard of troponin I level for cardiac involvement and then monitor how it changes with time and treatment.

\section{Methods}

19 Healthy volunteers were scanned at 1.5T. T1 (using a MOLLI sequence) and T2 mapping were performed in basal and mid short axis. The images were analysed by blinded readers to assess the reproducibility of the 12 segment myocardial segmentation approach.

Patients were recruited from the Rheumatology department which is a regional centre for myositis. Patients with cardiac symptoms or elevated troponin I were recruited. Recruits underwent CMR with tissue mapping and late gadolinium enhancement at the first visit with two subsequent non-contrast mapping studies followed by a final gadolinium enhanced CMR at 12 months.

All patient scans were performed on the same $1.5 \mathrm{~T}$ scanner and analysed by the same reader. In order to mitigate for bias a second reader, blinded to the clinical details including the troponin, repeated the analysis on $50 \%$ of studies. The T1 and T2 were calculated for 12 segments in each scan.

\section{Results}

Reproducibility of a 12 segment model for analysing tissue mapping sequences is excellent with a coefficient of variability of $1.4 \%$ for T1 and $2.6 \%$ for T2. Myositis patients with elevated troponin I had a significantly higher mean T1 and T2 than healthy volunteers $(p<0.0001$ both). Myositis patients with elevated troponin i have a significantly higher T1 and T2 than myositis patients with normal troponin I ( $p<0.01$ both). Patients with myositis and a negative troponin I have a significantly higher T1 and T2 than healthy volunteers (T1 p $<0.01, T 2 \mathrm{p}<0.05)$. There is a significant reduction in T1 and T2 values in patients with treatment over 8 months $(\mathrm{p}<0.05$ both).

\section{Conclusions}

A twelve segment model for myocardial T1 and T2 assessment is highly reproducible which is important in this disease process as there can be either focal or diffuse myocardial involvement. T1 and T2 are strongly associated with troponin I positivity with both likely to be a reflection of active inflammation in this disease process and improve with immune suppression therapy fairly quickly. 

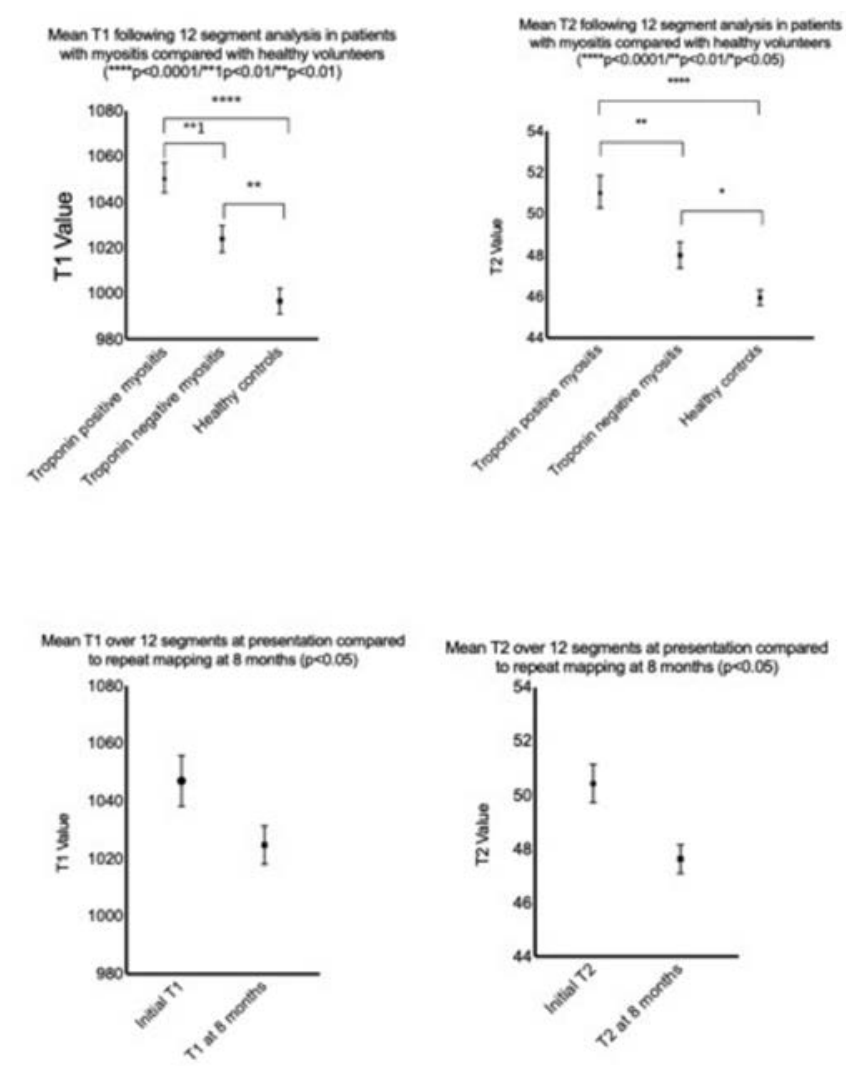

(c) Author(s) (or their employer(s)) 2020. No commercial re-use. See rights and permissions. Published by BMJ. Heart 2020;106:e1. doi:10.1136/heartjnl-2019-BSCMR.8corr1

(A) Check for updates 